\title{
L'annuaire des PTT comme moyen d'investigation géographique
}

Jean-Luc Piveteau

Autant qu'un signe, le téléphone est un moyen de puissance. Sa géographie se profile donc sur deux plans, l'un statique, l'autre dynamique: elle reflète une certaine structuration de l'espace existant; elle donne la mesure d'activités régionales différenciées, avec des pôles de croissance et des secteurs à développement lent.

Au service de cette double investigation généralement confondue, l'on exploite les informations qui concernent le trafic des conversations. L'analyse conduit à en distinguer deux aspects complémentaires. Le volume des communications données et reçues par les diverses agglomérations permet de classer ces dernières selon une certaine hiérarchie (cette hiérarchie urbaine dont les approches multiples restent toujours tangentes à la réalité). L'orientation des flux, second aspect majeur, précise les lignes de force de ces relations d'interdépendance par l'indication des «tropismes» de chaque localité.

Malheureusement, la documentation statistique des PTT, pour abondante, rigoureuse, et, à tant d'égards primordiale qu'elle soit, ne fait connaître, en détail, ni les volumes ni les flux. L'administration des téléphones n'effectue pas systématiquement la mesure des seconds. Elle ne l'entreprend qu'en cas de besoin, lorsque, dans un secteur particulier, l'encombrement des lignes appelle un réaménagement des circuits. Quant aux volumes, comptés eux, au contraire, mensuellement, ils ne s'appliquent pour ainsi dire jamais à une seule localité à la fois. La circonscription élémẹtaire, le «réseau local», comprend dans la généralité des cas plusieurs communes d'importance démographique et économique différente. La structure complexe des connections téléphoniques s'oppose à des dénombrements inférieurs à cette unité administrative. Au surplus, pour les agglomérations de quelque importance - elles jouent toutes un rôle de relais téléphonique: central nodal, central terminal interurbain, central de concentration interurbaine - les mêmes difficultés techniques font obstacle à la distinction entre le trafic local, c'est-à-dire entrant dans et sortant de la ville même, et le trafic de transit.

Le recours à l'annuaire des abonnés, assurément, ne peut en rien combler les lacunes touchant notre connaissance des flux au niveau de la localité. Par contre, et là est la justification de cet article, il per- met d'acquérir une image spatiale plus fine de l'équipement téléphonique, avec tout ce que celui-ci recèle de signification.

\section{Un instrument d'approche de contenu urbain}

C'est toutefois par un autre aspect de son utilisation que nous commencerons.

Les services des PTT n'ont jamais cherché, à notre connaissance, à établir une quelconque typologie de leurs clients. Ils ignorent la part d'équipement et celle du trafic qui reviennent à chaque grand groupe économique ou social. L'annuaire comble en grande partie ce vide: l'indication qu'il nous donne de l'activité de chaque abonné nous aide à définir les contours d'un support socio-économique de l'emploi du téléphone. Au surplus, à satisfaire cette curiosité sociologique, nous prenons la mesure de l'annuaire considéré comme instrument géographique servant à l'approche du contenu urbain ou villageois. Sous ce rapport, en effet, il atteindra une représentativité variable selon que les abonnements se répartiront plus ou moins proportionnellement à chaque catégorie de la population, ou selon qu'ils demeureront - dans une inégale distribution - plus ou moins constants d'une localité à l'autre.

La démarche que nous avons suivie est, dans son principe, simple. Nous avons confronté les différents groupes socio-professionnels de l'annuaire avec ceux du recensement civil - ce dernier servant évidemment de référence 1 . Le travail, pourtant, fut long et délicat. Long, car nous avons utilisé un mode de classement encore peu usuel, qui n'a jamais fait l'objet de récapitulations officielles: il nous a fallu procéder fiche par fiche, abonné par abonné. La division ternaire des statistiques fédérales nous est en effet apparue beaucoup trop sommaire pour le but que nous poursuivions. Nous avons adopté, moyennant de légères retouches, les six rubriques distinguées par MM. R. Girod et J.-F. Rouiller2:

1. classe dirigeante (professions libérales, haute finance, chefs de grandes entreprises, universitaires, etc. . . .); 2. classe moyenne - nous l'avons subdivisée en "indépendants» d'une part, avec principalement les artisans et les commerçants, et «agri- 
culteurs» d'autre part; 3. cadres; 4. employés; 5. ouvriers qualifiés et semi qualifiés; 6 . travailleurs subalternes, groupés sous le vocable de «manœuvres». Ces clivages reflètent avant tout une certaine hiérarchie sociale, fondée sur le revenu, le degré de formation, les responsabilités confiées, la marge d'autonomie. Mais il s'y intègre des notions de genre de vie propre à chaque catégorie, et, dans une moindre mesure, les distinctions économiques classiques (agriculteurs, ouvriers, employés, cadres).

$\mathrm{Si}$, dans la majorité des cas, la ventilation des professions s'effectue sans guère d'hésitation, elle prête encore trop souvent à ambiguité. Telle personne qui s'intitule «menuisier» entre-t-elle dans la catégorie des artisans, ou - ce qui, vérification faite, est le plus fréquent - dans celle des ouvriers spécialisés? Doit-on ranger un "directeur» sans autre épithète déterminante parmi les dirigeants ou les indépendants? Tel "fonctionnaire fédérale» parmi les «cadres» ou les «employés»? Et ainsi de suite. Les choix délicats ne manquent pas.

En outre, une fraction variable des abonnés n'indique pas de profession. La proportion change selon les communes: de 0 à $35 \%$, la moyenne se situant aux alentours de $15 \%$. Omission contrariante, car elle diminue la valeur significative de l'annuaire. Il faut se garder, toutefois, de surévaluer la déperdition d'informations. Une bonne part de ces abonnés correspond à des «inactifs» au sens classique du terme: des veuves sans métier, beaucoup de retraités. Un nombre plus restreint correspond aux gens qui jugent préférable de ne pas s'identifier professionnellement: ils se recrutent, semble-t-il, aux deux extrêmes de l'échelle sociale. Les détenteurs de résidences secondaires, enfin, constituent un troisième groupe, important dans les localités à vocation touristique, négligeable ailleurs.

Ces faiblesses délimitées, il n'en demeure pas moins possible de dégager plusieurs constantes. Les «dirigeants» ont, dans leur quasi-totalité, le téléphone; à l'inverse, les "manœuvres» en sont dépourvus. La proportion des abonnés oscille, autour de $15 \%$, en milieu ouvrier. Elle est plus forte chez les cadres que chez les employés, et chez les indépendants que chez ces derniers (5 cas sur 6 ). Ces similitudes n'empêchent pas les diagrammes de conserver leur personnalité. Ils ne se superposent pas. Faut-il attribuer leurs divergences au hasard? Les mettre en relation avec la taille des localités choisies, ou avec les régions auxquelles ces localités appartiennent? Autant de questions qui requèreraient d'élargir d'abord la base d'information. Mais l'on peut avancer d'ores et déjà que si l'annuaire paraît représentatif du contenu socio-économique d'une région en ce qui concerne les classes dirigeantes (dans tous les pays, du moins, où le téléphone tient une place analogue à celle qu'il tient en Suisse), il semble ne l'être que trop inégalement d'une agglomération à une autre, à l'égard des catégories restantes, pour qu'on l'utilise plus qu'à titre indicatif.

\section{Un moyen de mesure de l'équipement téléphonique}

En revanche, il nous donne accès à une image fine de la distribution des équipements téléphoniques. $\mathrm{Ce}$ point de vue nous retiendra davantage.

Pour une ville, d'abord, il est possible d'établir à l'aide de l'annuaire la proportion des abonnés par rapport à la population de chaque quartier. En raison de ce qui a été dit quelques lignes plus haut, la carte que l'on en retire doit refléter une certaine réalité sociale.

Mais les appareils téléphoniques constituent autant de petits pôles de rayonnement ou de convergence - ou les deux à la fois - pour les échanges entre les hommes. Ils confèrent à leurs détenteurs des contacts autres que ceux qu'autorisent la correspondance écrite, les chemins de fer, l'automobile, etc. Le fait donc d'entrer, grâce au téléphone, dans un réseau de relations supplémentaires vaut au commerçant, à l'employé qui s'abonne, une polarité (émettrice-réceptrice) au moins virtuelle plus grande qu'à son homologue non-abonné dont le niveau de vie et le volume d'activités sont pourtant, au départ, identiques. La carte en question aura donc une valeur dynamique.

A l'intérieur d'une commune d'habitat non exclusivement concentré, en second lieu, l'annuaire nous renseigne sur le nombre d'abonnés par écart. Sans doute cela ne préjuge-t-il en rien du trafic existant. Mais, rapporté à la population des différents hameaux ou fermes isolées quand on peut la connaître - ou, dans une approximation commode mais grossière, au nombre des maisons -, le chiffre des postes téléphoniques suggère un aspect important de la lutte contre l'isolement. Une comparaison entre différentes régions d'habitat dispersé donnerait tout son sens à un tel dénombrement.

Dans le cadre d'un réseau local, enfin et surtout, l'annuaire nous permet certaines analyses riches d'intérêt géographique.

Grâce à lui nous pouvons évaluer localité par localité, et à une faible marge d'erreur, le nombre des détenteurs de téléphone; autrement dit, «l'équipement» micro-régional; en d'autres termes encore, une certaine mesure du potentiel des relations téléphoniques.

L'opération paraît simple puisqu'elle consiste à dénombrer les abonnés - particuliers, entreprises, services publics - inscrits sous le vocable de chaque commune. En réalité, des précautions s'imposent. 


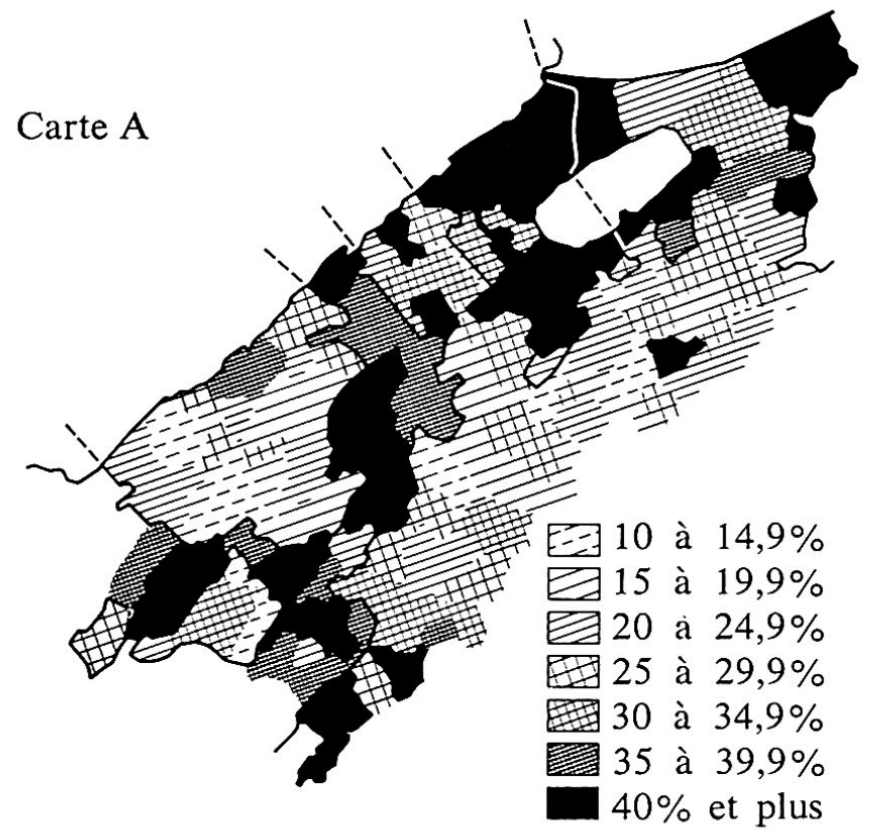

Pourcentage des abonnés par rapport à la population active d'après l'annuaire des PTT.

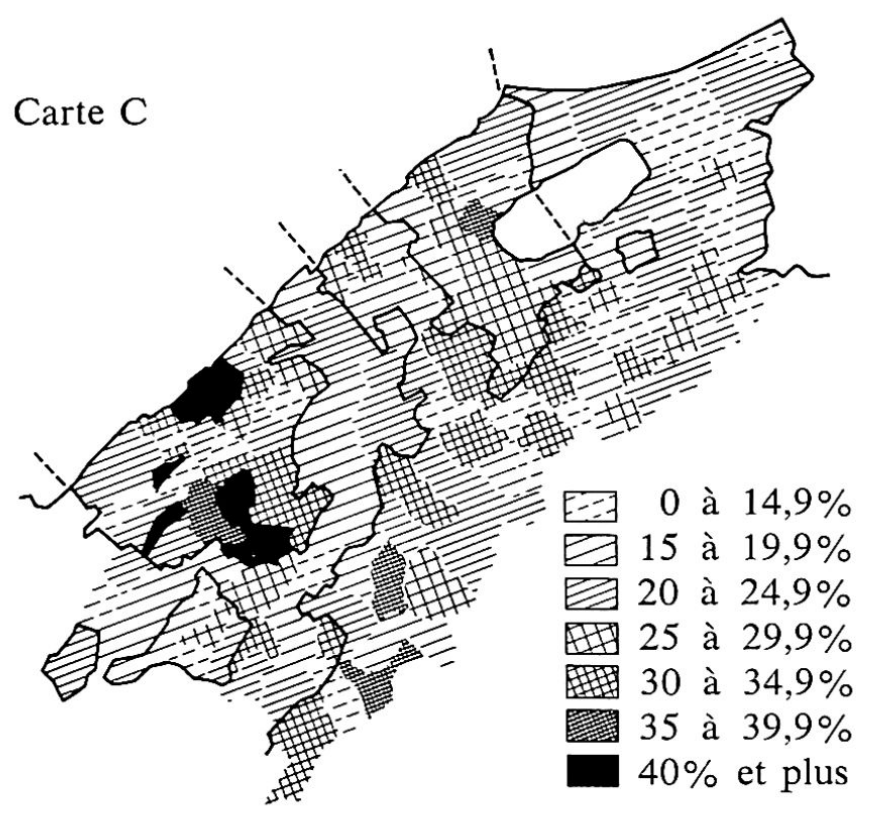

Pourcentage des abonnés par rapport à la population active d'après les statistiques des PTT.

Le rapprochement des deux cartes $B$ et $C$ permet de mesurer la précision qu'apporte l'emploi de l'annuaire dans la connaissance détaillée de la répartition des équipements.

1. Les écarts ne figurent pas sous le nom de la commune dont ils font partie, mais à leur place alphabétique: ce qui représentait pour nous une source d'informations non négligeable il y a un instant, devient cause de complications. Il faut ajouter ces abonnés dispersés à ceux du chef-lieu de la commune, si l'on veut effectuer des confrontations sans équivoque avec les chiffres de population qu'indiquent les recensements officiels.

2. Un même abonné apparaît souvent plusieurs fois: sous son nom; sous une rubrique professionnelle; sous plusieurs rubriques professionnelles de sens identique et de formulation différente (notamment quand il songe à se servir de l'annuaire comme d'un instrument de publicité); et, parfois, sous d'autres postes sans liens avec sa profession: tel est le cas des gens qui, à leur occupation principale, adjoignent celles de chef de section, commandant du feu, officier d'état civil, président d'une association ou autres.

Pour dépister ces répétitions ${ }^{3}$ qui gonfleraient indûment les statistiques, un travail minutieux d'archiviste s'impose. L'on pourrait s'en contenter. Il est préférable, toutefois, d'opérer un contrôle. Les PTT fournissent le chiffre global des abonnés d'un réseau local ainsi que les localités composant ce réseau. Il suffit donc d'additionner tous les abonnés qu'on a relevé dans ces dernières et d'en comparer le total au total officiellement indiqué pour le réseau local.
Le report sur carte de ces dénombrements fournit une première idée de l'équipement téléphonique détaillé. Peut-être même y trouve-t-on une réplique de ce que donnerait la cartographie du trafic. Celle-ci étant techniquement irréalisable au niveau communal, l'hypothèse demeure invérifiable. En revanche, un rapprochement entre le nombre des abonnés et la population de chaque commune - ou, comme nous l'avons préféré, de la population active - accroît considérablement la signification de cette étude de l'équipement. A titre d'expérience, nous avons examiné un ensemble contigu de 143 communes, situé dans la zone médiane du Plateau, sur la frange orientale du lac de Neuchâtel. Relativement homogène par ses conditions physiques (encore que les aptitudes naturelles inclinent la partie nord à se spécialiser dans la viticulture et le maraîchage), par son faible dynamisme démographique, par son armature de petites villes, cette région ne l'est plus sous l'angle linguistique, administratif et religieux: française au sud, allemande au nord, capricieusement partagée entre trois cantons, une marquetterie confessionnelle plus irrégulière encore, confirme en elle la forte empreinte de l'histoire sur la géographie humaine.

De la carte que nous avons dressée, se dégagent, à l'analyse, un certain nombre de traits (carte A).

1. L'équipement téléphonique ne se montre pas proportionnel à la population active. Il varie même dans une mesure sensible, allant de moins de $10 \%$ pour 8 communes à plus de $40 \%$ pour 33 autres. 


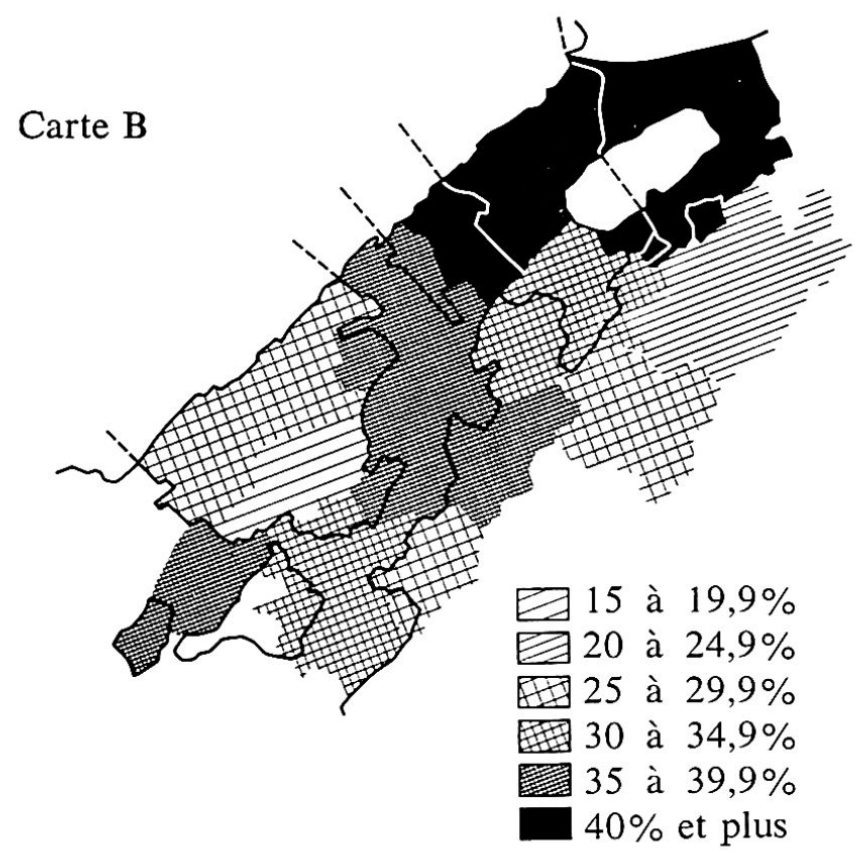

Pourcentage des «grands usagers» présumés par rapport au nombre d'abonnés.

2. La répartition des communes bien équipées est à mettre en relation avec l'importance des secteurs secondaire et tertiaire. La correspondance entre les taches de forte densité et les agglomérations de type urbain (Romont, Estavayer, Payerne, Avenches, Morat, Kerzers) la justifie. Cette corrélation, pourtant, n'explique pas tout.

3. Il faut faire intervenir des phénomènes de tourisme, de toute évidence, pour les rivages des lacs de Neuchâtel et de Morat (Chevroux à Cudrefin, Mur à Bellerive ... .).

4. Il semble, enfin, qu'on ne puisse se dispenser de faire appel à un état d'esprit général de la population, cause et conséquence d'un certain dynamisme économique, pour expliquer cette coïncidence que l'on observe, par exemple, entre le territoire vaudois et les secteurs d'équipements plus poussés.

Il était intéressant de savoir si une corrélation existait entre ce niveau d'équipement téléphonique et l'importance des agglomérations ${ }^{4}$. Nous avons donc construit un graphic dans lequel les coordonnées de chaque commune sont: en ordonnée, le chiffre de leur population active; en abscisse, le pourcentage d'abonnés par rapport à cette population active. Le résultat ${ }^{5}$ montre une forte dispersion. Il n'y a donc pas de relation simple de cause à effet entre l'importance des communes et leur équipement. A taille très inégale, deux localités peuvent accuser une même proportion d'abonnés. Un autre graphique mettant en rapport les pourcentages d'abonnés avec les communes regroupées, cette fois-ci, en 7 tranches de grandeurs différentes. Il montre ${ }^{5}$ une légère croissance d'ensemble de l'indice d'équipement en fonction de la dimension des localités. A deux anomalies près. L'une concerne le groupe des 42 communes qui rassemblent de 50 à 100 personnes actives. Le pourcentage d'abonnés y est sensiblement plus fort $(32 \%)$ que dans les groupes immédiatement supérieur et inférieur (27\%): est-ce là un pur hasard? L'autre concerne le fléchissement observé pour Payerne. L'indice d'équipement n'y atteint pas, de loin, celui de Morat ( $46 \%$ contre $58 \%$ ), alors que le peuplement y approche du double. Si l'on tient le chiffre de la population active pour un critère de la centralité d'une ville - et l'hypothèse paraît raisonnable l'on doit en conclure que l'équipement téléphonique ne croît pas nécessairement avec la centralité; si l'on part de cette autre idée quasi-certaine - elle ne contredit pas d'ailleurs la précédente - , selon laquelle l'équipement téléphonique constitue un facteur de centralité, il apparaît, en l'occurrence, que Payerne est moins polarisant que Morat. Signe d'un moindre dynamisme? Reflet d'une composition professionnelle dans laquelle ouvriers, employés et manœuvres - c'est-à-dire les catégories mal pourvues, en général, en téléphone - tiennent une place numériquement supérieure?

Quoiqu'il en soit de ces hypothèses, les graphiques, et la carte surtout, mettent en évidence une double structuration simultanée de l'espace: selon le mode hiérarchique et selon un mode continu. L'articulation microrégionale s'opère suivant une architecture de type "moléculaire» et suivant le principe de la tâche d'huile. Un même phénomène donc ici celui de l'équipement téléphonique - peut engendrer concurremment des espaces polarisés et des espaces homogènes.

\section{Un indicateur qualitatif de l'équipement téléphonique}

On peut pousser plus loin encore l'analyse de l'équipement téléphonique. Par la mention qu'il porte des professions, l'annuaire nous offre l'occasion de distinguer avec vraisemblance deux catégories d'abonnés: ceux qui emploient peu le téléphone, et ceux qui en usent beaucoup. Avec vraisemblance, non avec exactitude. Le nombre des communications échangées dépend en effet pour une part du caractère de chacun. Et cela nous échappe. Nous ne pouvons pas non plus deviner l'étendue des relations d'un individu sur la seule indication de son métier ni apprécier à la seule lecture de sa raison sociale le rayonnement commercial d'une entreprise. Enfin, le barême que nous avons appliqué, s'il nous paraît plausible, n'est 
fondé que sur des intuitions et non pas, faute d'enquête des PTT sur ce point, sur des comptages. Cette triple occasion d'erreurs nous a posé un problème de conscience. Vaut-il mieux laisser inexploitée une source d'information aussi conjecturale? Peut-on, au contraire, tirer profit, sous forme de suggestions, de données dont on ne sait au juste quelle marge d'incertitude les enveloppe? Nous avons opté pour la seconde solution, pensant que s'ils ne sont présentés qu'à titre d'hypothèses, les résultats obtenus stimuleront la recherche sans guère de risque de gauchir la vérité.

Dans la catégorie inférieure, celle des usagers présumés faibles ou moyens, nous avons rangé: les retraités, les employés, les ouvriers, les personnes sans signalement professionnel, les membres de l'enseignement, les agriculteurs, les artisans, les petits commerçants - pour ne citer que les principales rubriques. A la catégorie supérieure des abonnés présumés moyens ou grands usagers, nous avons rattaché les professions libérales (médecins, avocats, notaires, architectes ...), les administrations et les services publics (postes, chemins de fer, gendarmerie, etc.) et toutes les entreprises commerciales ou industrielles non artisanales. Il s'agit là, on le voit, d'un classement qui n'a rien de commun avec les grandes divisions économiques traditionnelles, ni même la structure socio-professionnelle utilisée plus haut.

Quel parti tirer de ce classement dualiste?

Il y a une tentation, dès l'abord, à écarter. Celle qui consisterait à évaluer le trafic téléphonique qui revient à chaque commune, en adoptant le chiffre des abonnés de la localité, moyennant l'affectation d'un certain coefficient (supérieur à l'unité) aux grands usagers. Les différences qui subsistent à l'intérieur de cette catégorie interdisent d'atteindre une précision suffisante.

Il faut s'en tenir, moins ambitieusement, à rapporter le nombre des "grands usagers» au nombre total d'abonnés par commune. On constate alors que le quotient varie sensiblement d'une localité à l'autre. Ce qui nous conduit à parler d'un degré variable de concentration - ou si l'on préfère de diffusion - pour chaque pôle de trafic téléphonique. Les agglomérations à trafic "concentré» seront les villes, villages ou hameaux pour lesquels la proportion des grands usagers sera forte - quelle que soit l'intensité du trafic lui même. Les agglomérations à trafic diffus seront, à l'inverse, celles pour lesquelles la proportion des faibles usagers sera grande: peu ou très abondantes, les communi- cations téléphoniques s'y répartiront entre un plus grand nombre d'abonnés.

Plusieurs petites communes rurales accusent une concentration élevée, ce qui peut s'expliquer par un faible équipement général. Presque seules, en effet, dans ce cas, les professions tenues à avoir le téléphone en sont pourvues. Mais le rapport ne faiblit pas dans d'autres communes, elles, bien fournies en postes. La cause, ici, doit être recherchée dans la présence d'un nombre élevé d'activités "grandes usagères» par rapport à la population totale. Autrement dit, dans ce second cas, ce n'est pas à un effectif important "d'actifs» sans téléphone que l'on doit le fort indice de concentration, mais à la multiplication des professions "dynamiques» au sein de la population active. Les agglomérations urbaines montrent des contrastes analogues. Il semble toutefois que leur indice décroisse en sens inverse de leur trafic - pour autant qu'on puisse supposer que celui-ci se calque grossièrement sur le nombre d'habitants - : Payerne a moins de "grands usagers» que Morat, Morat qu'Avenches, Avenches qu'Estavayer (Romont déroge à la règle). Faut-il imputer ce phénomène à une représentation plus grande d'ouvriers et d'employés parmi les abonnés des plus grandes localités 6 ?

A l'étude de l'équipement proprement dit et sans se substituer à celle du volume total des communications entrantes et sortantes enregistrées en chaque lieu, cette mesure de la "concentration» présumée du trafic a donc l'intérêt d'ajouter une nuance qualitative. Mais autant la carte de répartition des équipements invitait à distinguer quelques petites unités homogènes, autant celle des quotients de concentration s'y prête mal (carte C). Signe de la complexité des phénomènes de relations téléphoniques, cette marquetterie est peut-être aussi l'indice qu'à l'échelle micro-régionale on n'atteint pas toujours à autant de relative simplicité dans l'organisation des liens d'interdépendance qu'à celle des grands ensembles régionaux.

Malgré la lenteur de son dépouillement et en dépit de ses ambiguités, l'annuaire permet donc une approche détaillée de l'aspect le plus subtil de la géographie des relations, et par de là, livre des aperçus sur la trame fine de l'espace humain. Il donne, ce faisant, un avant-goût de tout ce que révèlerait une exploitation mécanographique des factures payées chaque année par les usagers du téléphone. Aussi longtemps que cette dernière entreprise, pour des raisons pécuniaires principalement, nous demeurera inaccessible, il conservera un précieux rôle de suggestion au service de l'investigation régionale. 
1 Afin d'obtenir des comparaisons valables avec les chiffres du recensement fédéral de décembre 1960, nous avons utilisé l'édition «1961-62» de l'annuaire, qui correspond à une situation téléphonique sensiblement contemporaine (fin novembre 1960 à février 1961).

2 R. Girod et J.-F. Rouiller: «Milieu social et orientation de la carrière des adolescents» fasc. I, Genève, 1963 .

3 L'erreur contraire, imputable aux personnes qui refusent de laisser inscrire leur nom dans l'annuaire, est négligeable.

4 L'indice de groupement élevé de l'habitat, dans cette partie du pays, permet une assimilation des effectifs de la commune avec ceux de son chef-lieu.

5 Ces graphiques ont été présentés devant la section de géographie de la S.H.S.N. au congrès de septembre 1965 .

6 Ceci ne contredit nullement l'hypothèse de la page 4 , lignes 42 et suivantes.
Das Telephonabonnenten-Verzeichnis der PTT als geographisches Forschungsmittel

Der Telephonverkehr interessiert den Geographen in doppelter Hinsicht: einerseits als Phänomen der menschlichen Beziehungen, anderseits als Zeichen und Faktor der Zentralität beim Studium des städtischen Gefüges.

Die von der PTT gelieferten statistischen Unterlagen bleiben unersetzlich, was Umfang, Richtung und Weite des Telephonverkehrs betrifft. Aber das Abonnentenverzeichnis stellt eine Informationsergänzung dar, welche in einigen Beziehungen "geographischer» ist als die PTT-Statistik. In der Tat ist es bis jetzt das einzige Mittel, den sozio-ökonomischen und mikro-regionalen Telephonverkehr mit einer gewissen Genauigkeit zu analysieren. Es erlaubt, durch Vergleich mit den offiziellen Zählungen, jene sozio-professionellen Kategorien kennenzulernen, welche den größten Teil des Telephonverkehrs ausmachen. Ferner gestattet es, die Verteilung des Telephons einer Region nach Stadtquartieren, nach Orten innerhalb einer Gemeinde und vor allem gemeindeweise im Rahmen des «Ortsnetzes» - wie dies die PTT definiert — zu beurteilen. Diese Verteilung, bezogen auf die aktive Bevölkerung und die Verkehrsvermutungen, welche die Berufseintragungen der Abonnenten liefern, regt $\mathrm{zu}$ verschiedenen Arbeitshypothesen hinsichtlich der Raumgliederung und der vielseitigen Korrelationen an, die zwischen der Ortschaftengröße und ihrer Polarität bestehen. 